\title{
Alcohol-Induced Sinus Bradycardia and Hypotension in Patients with Syncope
}

\author{
Masato Tsutsur, M.D., Takeyuki Matsuguchi, M.D., \\ Hiroyuki Tsutsui, M.D., Tetsuhiko Yoshida, M.D.,* \\ Shingo Yoshihara, M.D., Kunihiko Yamamoto, M.D., \\ Ryuichi Hrsanou, M.D., * Hiroaki Shimokawa, M.D., \\ and Shuichi Okamatsu, M.D.
}

\section{SUMmary}

We observed 2 cases of repeated episodes of syncope after alcohol ingestion. Both patients were light drinkers and had carotid sinus hypersensitivity. In both cases, alcohol loading tests repeatedly induced sinus bradycardia and hypotension 1.0-1.5 hours after drinking alcohol. Atropine was effective in improving symptoms. A loading test using a glucose solution of equivalent osmolarity and volume was negative. Acute alcohol ingestion usually increases heart rate with variable effects on blood pressure. However, our 2 cases exhibited unusual alcoholinduced sinus bradycardia and hypotension, suggesting a paradoxical increase in parasympathetic activity and/or decrease in sympathetic activity.

\section{Key Words:}

Alcohol Bradycardia Hypotension Syncope

$\mathrm{T}$ HE hemodynamic effects of alcohol have long been recognized and extensively studied. In contrast to alcohol's variable effects on blood pressure, almost all studies have shown an increase in heart rate. ${ }^{1)-6}$ ) This effect is probably due to a reflex response to maintain blood pressure after arteriovenous dilatation (a direct effect of ethanol) and/or volume depletion (a diuretic effect of ethanol). ${ }^{1} \quad$ In some cases acute alcohol ingestion induces orthostatic hypotension and syncope due to those direct and/or diuretic effects of ethanol. ${ }^{6}$ However, among the 113 cases of syncope seen in our hospitals in the past 5 years, 2 cases exhibited unusual sinus bradycardia and hypotension induced by alcohol ingestion. We report here these 2 cases of unusual alcohol-induced marked sinus bradycardia and hypotension.

From the Department of Cardiology, Iizuka Hospital, Iizuka, Fukuoka and the Department of Cardiology, Saga Prefectural Hospital,* Saga, Japan.

Address for correspondence: Masato Tsutsui, M.D., Department of Cardiology, Iizuka Hospital, 3-83 Yoshio-machi, Iizuka 820, Fukuoka, Japan.

Received for publication March 2, 1992.

Accepted July 1, 1992. 


\section{Gase Report}

Case 1: A 55-year-old man was admitted to our hospital because of three syncopal attacks without nausea or vomiting after drinking alcohol. He had a 3-year history of mild diabetes mellitus with no organ involvement, which was well controlled with diet therapy alone. Electrocardiogram (EGG) and brain computerized tomography were normal. Two-dimensional and Doppler echocardiograms were normal. Holter ambulatory ECG showed no serious arrhythmias. After massage of the right carotid sinus, heart rate (HR) decreased from 62 to about $20 \mathrm{bpm}$ and systolic blood pressure (SBP) fell from 115 to $64 \mathrm{mmHg}$; at that time he complained of discomfort. Cold pressor and tilt tests were normal.

In the alcohol loading test (Fig. 1), the patient drank $180 \mathrm{ml}$ of Japanese sake (equivalent to $29 \mathrm{ml}$ of ethanol) over $10 \mathrm{~min}$. He was in a fasting state and supine posture. HR began to increase rapidly after the beginning of the test, reached $102 \mathrm{bpm}$ after $20 \mathrm{~min}$, and then gradually decreased. SBP did not change immediately after alcohol ingestion, but decreased by $20-30 \mathrm{mmHg}$ after $30 \mathrm{~min}$. Eighty-five min after the start of the test, HR abruptly decreased to $38 \mathrm{bpm}$ and SBP fell to $64 \mathrm{mmHg}$, and after 1 min nausea and vomiting occurred without syncope (Fig. 1). Atropine $1 \mathrm{mg}$ was injected intravenously and $\mathrm{HR}$ immediately increased to $95 \mathrm{bpm}$

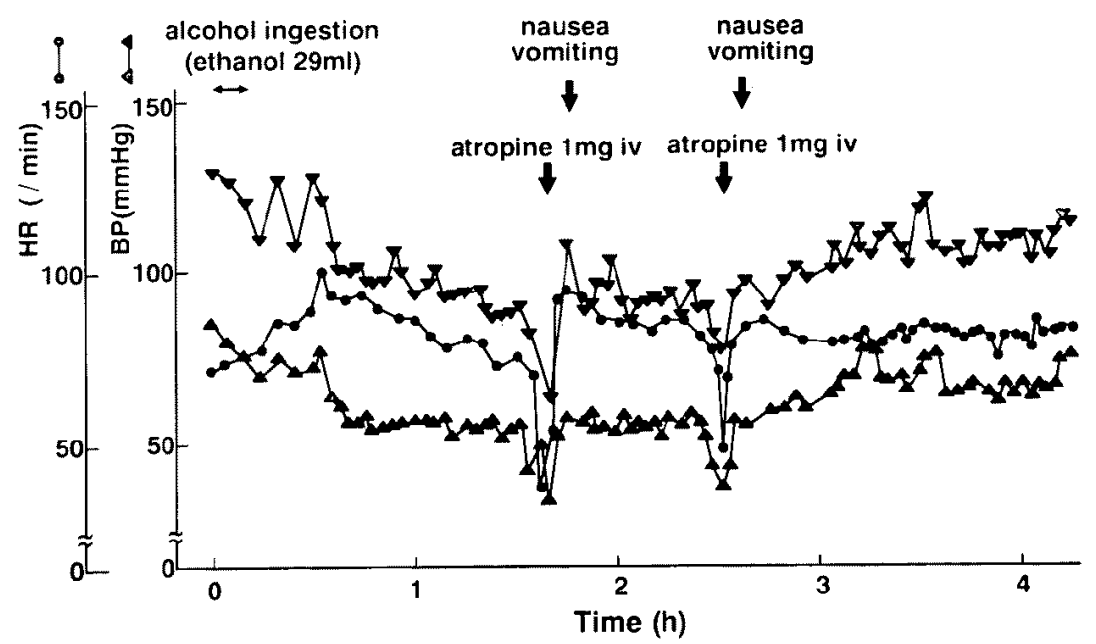

Fig. 1. Case 1. At $85 \mathrm{~min}$ after starting the alcohol loading test, sinus bradycardia (HR $38 \mathrm{bpm}$ ) and hypotension (SBP $64 \mathrm{mmHg}$ ) were provoked abruptly, followed by nausea and vomiting without syncope. After $135 \mathrm{~min}$, the same attack occurred again. Atropine administration was effective in reversing the hemodynamic changes and improving the symptoms. 
and SBP recovered to $110 \mathrm{mmHg}$, following which the symptoms subsided. After 135 min the same hemodynamic changes occurred, followed by nausea and vomiting but disappeared again after administration of atropine. Serum concentrations of ethanol and acetaldehyde during the first attack increased from 0.1 to $3.3 \mathrm{mM}$ (normal range; less than $2.2 \mathrm{mM}$ ) and from 2.4 to $37.9 \mu \mathrm{M}$ (normal range; less than $5.0 \mu \mathrm{M}$ ), respectively. These concentrations remained at high levels during the second attack $(3.7 \mathrm{mM}$ and $10.0 \mu \mathrm{M}$, respectively). On another day, the alcohol loading test was repeated in the cardiac catheterization laboratory and the same attack was induced. During the attack, there was no significant ST-T change on ECG and coronary angiograms showed no significant hyperconstriction of the coronary arteries. The loading test using a glucose solution of equivalent osmolarity and volume to the sake alcohol did not induce bradycardia, hypotension or syncope.

Case 2: A 65-year-old man experienced syncopal attacks twice without nausca or vomiting after acute consumption of alcohol. Physical examination showed that blood pressure was $150 / 70 \mathrm{mmHg}$ but otherwise normal. ECG and electroencephalogram were normal. Two-dimensional and Doppler echocardiograms revealed mild left ventricular hypertrophy without left ventricular outflow tract obstruction. Holter ambulatory ECG

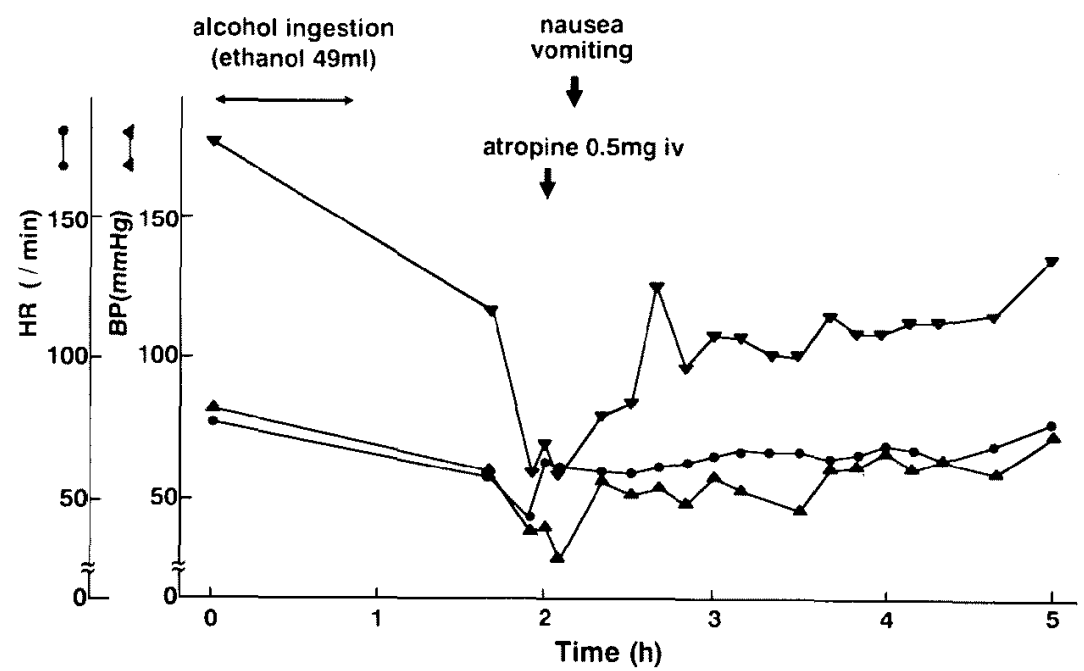

Fig. 2. Case 2. At 65 min after starting the loading test, sinus bradycardia (HR $44 \mathrm{bpm}$ ) and hypotension (SBP $60 \mathrm{mmHg}$ ) were provoked and shortly after that the patient complained of nausea and romiting abruptly without syncope. Atropine administration was effective in reversing the hemodynamic changes and improving the symptoms. 
showed no serious arrhythmias. By massaging the right carotid sinus, sinus arrest ( $\max R-R$ interval $2.8 \mathrm{sec}$ ) and hypotension (SBP fell from 118 to $64 \mathrm{mmHg}$ ) were provoked. Cold pressor and tilt tests were normal.

In the alcohol loading test (Fig. 2), the patient drank $600 \mathrm{ml}$ of beer and $100 \mathrm{ml}$ of shochu (a type of Japanese whiskey, total ethanol content $49 \mathrm{ml}$ ) over $50 \mathrm{~min}$ in a fasting state and in the supine position. After 65 min, HR decreased to $44 \mathrm{bpm}$ and SBP fell to $60 \mathrm{mmHg}$ and shortly after that the patient complained of nausea and vomited abruptly without syncope. There was no significant ST-T change on ECG. After intravenous injection of $0.5 \mathrm{mg}$ of atropine and intravenous drip infusion of $0.9 \%$ sodium chloride solution, HR and SBP gradually returned to baseline levels and the symptoms subsided. The loading test using a glucose solution of equivalent osmolarity and volume to the alcohol mixture used in the alcohol loading test induced no hemodynamic changes or syncope.

\section{Discussion}

We have demonstrated alcohol-induced sinus bradycardia and hypotension in 2 patients with syncope. To our knowledge such cases have not been reported previously. These 2 cases have the following clinically distinctive features: 1) carotid sinus hypersensitivity (mixed type; cardioinhibitory and vasodepressor type), 2) light drinkers in case 1, serum acetaldehyde concentration was high suggesting the possibility of aldehyde dehydrogenase I deficiency, 3) bradycardia and hypotension were induced 1.0-1.5 hours after drinking alcohol, 4) intravenous injection of atropine was effective, and 5) the symptoms were reproducible.

In our 2 cases, the specific effect of ethanol appears to have been responsible for the hemodynamic changes and syncope since the loading test using a glucose solution of equivalent osmolarity and volume was negative. In addition, since nausea and vomiting were not present during the syncopal attacks in either patient and the hemodynamic changes preceded nausea and vomiting in the alcohol loading tests, vagotomy may not be the primary mechanism causing the hemodynamic changes and syncope. Vasodilatation (direct effect of ethanol) and/or volume depletion (diuretic effect of ethanol) may cause orthostatic hypotension and syncope. ${ }^{6)}$ Syncope after acute ethanol ingestion is seen particularly in association with micturition. ${ }^{\text {) }}$ However, in our 2 cases syncope occurred without micturition. There were no signs and symptoms of sinus nodal disease or autonomic nerve abnormalities. In case 1 sinus bradycardia and hypotension occurred twice. The second attack may also have been induced by alcohol or alcohol metabolite(s) 
because serum concentrations of ethanol and acetaldehyde during the second attack were still at high levels.

Acute alcohol ingestion generally inhibits the parasympathetic nervous system $^{51}$ or stimulates the sympathetic nervous system. ${ }^{6)}$ On the contrary, in the present 2 cases, it is suggested that alcohol or alcohol metabolite(s) paradoxically increased parasympathetic activity and/or decreased sympathetic activity, causing marked sinus bradycardia, hypotension and syncope. In these cases, atropine administration may be useful to improve symptoms. Alcohol intolerance and carotid sinus hypersensitivity may have been involved in the alcohol-induced hemodynamic changes and syncope in our 2 cases, however, the exact mechanism remains to be elucidated.

\section{REFERENGES}

1. Howes LG, Reid JL: The effect of alcohol on local, neural and humoral cardiovascular regulation. Clin Sci $71: 9,1986$

2. Ireland MA, Vandongen R, Davidson L, Beilin LJ, Rouse IL: Acute effects of moderate alcohol consumption on blood pressure and plasma catecholamines. Clin Sci 66: 643, 1984

3. Kupari M: Acute cardiovascular effects of ethanol. A controlled non-invasive study. $\mathrm{Br}$ Heart J 49: 174, 1983

4. Kettunen $\mathbf{R}$, Timisjarvi J, Saukkoh $\mathbf{P}$, Koskela $\mathbf{M}$ : Influence of ethanol on systemic and pulmonary hemodynamics in anesthetized dogs. Acta Physiol Scand 118: 209, 1983

5. Newlin DB, Byine EA, Porges SW: Vagal mediation of the effect of alcohol on heart rate. Alcoholism 14: 421, 1990

6. Jonson RH, Eisenhofer G, Lambie DG: The effect of acute and chronic ingestion of ethanol on the autonomic nervous system. Drug Alcohol Depend 18: 319, 1986 